\title{
Topological obstructions for submanifolds in low codimension
}

\author{
Christos-Raent Onti and Theodoros Vlachos
}

\begin{abstract}
We prove integral curvature bounds in terms of the Betti numbers for compact submanifolds of the Euclidean space with low codimension. As an application, we obtain topological obstructions for $\delta$-pinched immersions. Furthermore, we obtain intrinsic obstructions for minimal submanifolds in spheres with pinched second fundamental form.
\end{abstract}

\section{Introduction}

By the Nash's embedding theorem, every Riemannian manifold can be isometrically immersed into a Euclidean space with sufficiently high codimension. On the other hand, there are results that impose restrictions on isometric immersions with low codimension (cf. [10,18-21,23]). Most of these obstructions are pointwise conditions on the range of curvature. Here, we investigate obstructions for immersions with low codimension that involve total curvature. In particular, we are interested in the $L^{n / 2}$-norm of the $(0,4)$ tensor $R-(\mathrm{scal} / n(n-1)) R_{1}$, where $R$ and scal denote the $(0,4)$-curvature tensor and the scalar curvature of the induced metric $g$ respectively, and $R_{1}=(1 / 2) g \otimes g$, where $₫$ stands for the Kulkarni-Nomizu product. Shiohama and Xu [25] gave a lower bound in terms of the Betti numbers for compact hypersurfaces in the Euclidean space $\mathbb{R}^{n+1}$. For higher codimension, they raised the following

Problem. Let $M^{n}, n \geq 3$, be a compact $n$-dimensional Riemannian manifold which admits an isometric immersion into $\mathbb{R}^{2 n-1}$. Does there exist a constant $\varepsilon(n)$, depending only on $n$, such that if

$$
\int_{M^{n}}\left\|R-\frac{\text { scal }}{n(n-1)} R_{1}\right\|^{n / 2} d M<\varepsilon(n)
$$

then $M^{n}$ is homeomorphic to the sphere $\mathbb{S}^{n}$ ?

2010 Mathematics Subject Classification. Primary 53C40, 53C20; Secondary 53C42.

Key Words and Phrases. Curvature tensor, $L^{n / 2}$-norm of curvature, Betti numbers, $\delta$-pinched immersions, flat billinear forms, Weyl tensor. 
In the present paper, we provide integral curvature bounds in terms of the Betti numbers, for compact submanifolds of Euclidean space with low codimension. As a consequence, we obtain partial answers to the above problem and extend previous ones given in [30]. Throughout the paper, all manifolds under consideration are assumed to be without boundary, connected and oriented. Our main result is stated as follows.

Theorem 1. Given an integer $n \geq 4$ and $\delta \in(1 / n, 1)$, there exists a positive constant $c(n, \delta)$ such that if $M^{n}$ is a compact $n$-dimensional Riemannian manifold that admits an isometric immersion $f$ in $\mathbb{R}^{n+k}, 2 \leq k \leq n / 2$, then

$$
\int_{M^{n}}\left\|R-\frac{\mathrm{scal}}{n(n-1)} R_{1}\right\|^{n / 2} d M+\int_{M^{n}}\left(S-\delta n^{2} H^{2}\right)_{+}^{n / 2} d M \geq c(n, \delta) \sum_{i=k}^{n-k} \beta_{i}\left(M^{n} ; \mathbb{F}\right),
$$

where $S$ is the squared norm of second fundamental form, $H$ the mean curvature ${ }^{1}$ of $f$, $\left(S-\delta n^{2} H^{2}\right)_{+}=\max \left\{S-\delta n^{2} H^{2}, 0\right\}$ and $\beta_{i}\left(M^{n} ; \mathbb{F}\right)$ the $i$-th Betti number of $M^{n}$ over an arbitrary coefficient field $\mathbb{F}$. Furthermore,

(i) If

$$
\int_{M^{n}}\left\|R-\frac{\mathrm{scal}}{n(n-1)} R_{1}\right\|^{n / 2} d M+\int_{M^{n}}\left(S-\delta n^{2} H^{2}\right)_{+}^{n / 2} d M<c(n, \delta),
$$

then $M^{n}$ has the homotopy type of a $C W$-complex with no cells of dimension $i$ for $k \leq i \leq n-k$. Moreover, if $k=2$, then the fundamental group $\pi_{1}\left(M^{n}\right)$ is a free group on $\beta_{1}\left(M^{n} ; \mathbb{Z}\right)$ generators and if $\pi_{1}\left(M^{n}\right)$ is finite then $M^{n}$ is homeomorphic to $\mathbb{S}^{n}$.

(ii) If the scalar curvature of $M^{n}$ is everywhere non-positive, then

$$
\int_{M^{n}}\left\|R-\frac{\mathrm{scal}}{n(n-1)} R_{1}\right\|^{n / 2} d M+\int_{M^{n}}\left(S-\delta n^{2} H^{2}\right)^{n / 2} d M \geq c(n, \delta) \sum_{i=0}^{n} \beta_{i}\left(M^{n} ; \mathbb{F}\right) .
$$

(iii) If the scalar curvature is everywhere non-positive and

$$
\int_{M^{n}}\left\|R-\frac{\text { scal }}{n(n-1)} R_{1}\right\|^{n / 2} d M+\int_{M^{n}}\left(S-\delta n^{2} H^{2}\right)^{n / 2} d M<3 c(n, \delta)
$$

then $M^{n}$ is homeomorphic to $\mathbb{S}^{n}$.

In the case where (1) is satisfied, the homology groups of $M^{n}$ must satisfy the condition $H_{i}\left(M^{n} ; \mathbb{F}\right)=0$ for all $k \leq i \leq n-k$, where $\mathbb{F}$ is any coefficient field.

The idea of the proof is to relate the $L^{n / 2}$-norm of the tensor $R-(\operatorname{scal} / n(n-1)) R_{1}$ with the Betti numbers using Morse theory, Chern-Lashof results [11,12] and the Gauss

\footnotetext{
${ }^{1}$ The mean curvature is given by $H=|\mathcal{H}|$, where $\mathcal{H}$ denotes the mean curvature vector.
} 
equation. To this aim we prove an algebraic inequality for symmetric bilinear forms (see Prop. 9). The presence of the integral $\int_{M^{n}}\left(S-\delta n^{2} H^{2}\right)_{+}^{n / 2} d M$ in Theorem 1 is essential since the algebraic inequality fails by dropping the corresponding term.

The above integral measures how far an immersion deviates from being $\delta$-pinched. The latter means that the inequality $S \leq \delta n^{2} H^{2}$ holds everywhere, in which case $\delta \geq$ $1 / n$. We note that Shiohama and $\mathrm{Xu}[26,27]$ gave a topological lower bound of the above integral in the case where $\delta=1 / n$. The geometry and the topology of $\delta$-pinched immersions have been studied by several authors (see $[1,2,2,3,6-8]$ ) in the case where $\delta=$ $1 /(n-1)$. Our results provide information on $\delta$-pinched immersions for any $\delta \in(1 / n, 1)$. Indeed, the following corollary follows immediately from Theorem 1 and gives an intrinsic obstruction to $\delta$-pinched immersions.

Corollary 2. If a compact $n$-dimensional Riemannian manifold $M^{n}, n \geq 4$, admits an isometric $\delta$-pinched immersion in $\mathbb{R}^{n+k}, 2 \leq k \leq n / 2$, for some $\delta \in(1 / n, 1)$, then

$$
\int_{M^{n}}\left\|R-\frac{\mathrm{scal}}{n(n-1)} R_{1}\right\|^{n / 2} d M \geq c(n, \delta) \sum_{i=k}^{n-k} \beta_{i}\left(M^{n} ; \mathbb{F}\right) .
$$

In particular, if

$$
\int_{M^{n}}\left\|R-\frac{\text { scal }}{n(n-1)} R_{1}\right\|^{n / 2} d M<c(n, \delta),
$$

then $M^{n}$ has the homotopy type of a $C W$-complex with no cells of dimension $i$ for $k \leq$ $i \leq n-k$. Moreover, if $k=2$, then $\pi_{1}\left(M^{n}\right)$ is a free group on $\beta_{1}\left(M^{n} ; \mathbb{Z}\right)$ generators and if $\pi_{1}\left(M^{n}\right)$ is finite then $M^{n}$ is homeomorphic to $\mathbb{S}^{n}$.

The next results are easy consequences of Theorem 1 and provide partial answers to the problem raised by Shiohama and Xu.

Corollary 3. If a compact $n$-dimensional Riemannian manifold $M^{n}, n \geq 4$, admits an isometric immersion $f$ in $\mathbb{R}^{n+k}, 2 \leq k \leq n / 2$, such that

$$
\int_{M^{n}}\left\|R-\frac{\mathrm{scal}}{n(n-1)} R_{1}\right\|^{n / 2} d M<\lambda c(n, \delta)
$$

and

$$
\int_{M^{n}}\left(S-\delta n^{2} H^{2}\right)_{+}^{n / 2} d M \leq(1-\lambda) c(n, \delta) \sum_{i=k}^{n-k} \beta_{i}\left(M^{n} ; \mathbb{F}\right),
$$

where $\lambda \in(0,1)$ and $\delta \in(1 / n, 1)$, then $f$ is $\delta$-pinched and $M^{n}$ has the homotopy type of a $C W$-complex with no cells of dimension $i$ for $k \leq i \leq n-k$. Furthermore,

(i) If $k=2$, then $\pi_{1}\left(M^{n}\right)$ is a free group on $\beta_{1}\left(M^{n} ; \mathbb{Z}\right)$ generators and if $\pi_{1}\left(M^{n}\right)$ is finite then $M^{n}$ is homeomorphic to $\mathbb{S}^{n}$. 
(ii) If the mean curvature is everywhere positive and $\delta=1 /(n-1)$, then $M^{n}$ is diffeomorphic to $\mathbb{S}^{n}$.

Corollary 4. If a compact $n$-dimensional Riemannian manifold $M^{n}, n \geq 4$, admits an isometric immersion in $\mathbb{R}^{n+k}, 2 \leq k \leq n / 2$, such that

$$
\int_{M^{n}}\left\|R-\frac{\mathrm{scal}}{n(n-1)} R_{1}\right\|^{n / 2} d M \leq \lambda c(n, \delta) \sum_{i=k}^{n-k} \beta_{i}\left(M^{n} ; \mathbb{F}\right)
$$

and

$$
\int_{M^{n}}\left(S-\delta n^{2} H^{2}\right)_{+}^{n / 2} d M<(1-\lambda) c(n, \delta),
$$

where $\lambda \in(0,1)$ and $\delta \in(1 / n, 1)$, then $M^{n}$ is isometric to a constant curvature sphere.

Minimal submanifolds with pinched second fundamental form have been studied by Simons [28], Chern, do Carmo, Kobayashi [9] and Leung [16], among others. We provide intrinsic obstructions for minimal submanifolds in spheres with sufficiently pinched second fundamental form.

Corollary 5. Let $f: M^{n} \rightarrow \mathbb{S}^{n+k-1}, 2 \leq k \leq n / 2$, be an isometric minimal immersion of a compact $n$-dimensional Riemannian manifold $M^{n}, n \geq 4$. If the squared norm of the second fundamental form satisfies $S \leq n(\delta n-1)$ for some $\delta \in(1 / n, 1)$, then

$$
\int_{M^{n}}\left\|R-\frac{\mathrm{scal}}{n(n-1)} R_{1}\right\|^{n / 2} d M \geq c(n, \delta) \sum_{i=k}^{n-k} \beta_{i}\left(M^{n} ; \mathbb{F}\right) .
$$

In particular, if

$$
\int_{M^{n}}\left\|R-\frac{\text { scal }}{n(n-1)} R_{1}\right\|^{n / 2} d M<c(n, \delta),
$$

then $M^{n}$ has the homotopy type of a $C W$-complex with no cells of dimension $i$ for $k \leq$ $i \leq n-k$. Moreover, if $k=2$, then the fundamental group $\pi_{1}\left(M^{n}\right)$ is a free group on $\beta_{1}\left(M^{n} ; \mathbb{Z}\right)$ generators and if $\pi_{1}\left(M^{n}\right)$ is finite then $M^{n}$ is homeomorphic to $\mathbb{S}^{n}$.

It is well known that the Weyl tensor $\mathcal{W}$ of a $n$-dimensional Riemannian manifold $M^{n}, n \geq 4$, vanishes if and only if $M^{n}$ is conformally flat. The study of conformally flat manifolds, from the point of view of submanifold theory, was initiated by Cartan in [4]. The case of compact conformally flat hypersurfaces of Euclidean space has been studied by Do Carmo, Dajczer and Mercuri [13]. For low codimension $k$, Moore [19] proved that such submanifolds have the homotopy type of a CW-complex with no cells of dimension $i, k<i<n-k$. Therefore, it is natural to seek for restrictions on the topology of compact almost conformally flat submanifolds, in the sense that the Weyl tensor is sufficiently small in a suitable norm. 
The case of hypersurfaces has been treated in [2]. In this paper, we prove an inequality for the $L^{n / 2}$-norm of the Weyl tensor for compact $n$-dimensional Riemannian manifolds that allow conformal immersions in the Euclidean space with low codimension. As a consequence, we obtain a partial answer to the above question.

Theorem 6. Given $n \geq 6$ and $\delta \in(1 / n, 1)$, there exists a positive constant $c_{1}(n, \delta)$ such that if $M^{n}$ is a compact n-dimensional Riemannian manifold that admits a conformal immersion in $\mathbb{R}^{n+k}, 2 \leq k \leq[(n-2) / 2]$, then

$$
\int_{M^{n}}\|\mathcal{W}\|^{n / 2} d M+\int_{M^{n}}\left(S-\delta n^{2} H^{2}\right)_{+}^{n / 2} d M \geq c_{1}(n, \delta) \sum_{i=k+1}^{n-k-1} \beta_{i}\left(M^{n} ; \mathbb{F}\right)
$$

In particular, if

$$
\int_{M^{n}}\|\mathcal{W}\|^{n / 2} d M+\int_{M^{n}}\left(S-\delta n^{2} H^{2}\right)_{+}^{n / 2} d M<c_{1}(n, \delta),
$$

then $M^{n}$ has the homotopy type of a $C W$-complex with no cells of dimension $i$ for $k<$ $i<n-k$.

As an application of Theorem 6, we may obtain results similar to Corollaries 2-5 for the Weyl tensor instead of the tensor $R-(\operatorname{scal} / n(n-1)) R_{1}$. For instance, we have the following

Corollary 7. If a compact $n$-dimensional Riemannian manifold $M^{n}, n \geq 6$, admits a conformal $\delta$-pinched immersion in $\mathbb{R}^{n+k}, 2 \leq k \leq[(n-2) / 2]$, for some $\delta \in(1 / n, 1)$, then

$$
\int_{M^{n}}\|\mathcal{W}\|^{n / 2} d M \geq c_{1}(n, \delta) \sum_{i=k+1}^{n-k-1} \beta_{i}\left(M^{n} ; \mathbb{F}\right)
$$

In particular, if

$$
\int_{M^{n}}\|\mathcal{W}\|^{n / 2} d M<c_{1}(n, \delta)
$$

then $M^{n}$ has the homotopy type of a $C W$-complex with no cells of dimension $i$ for $k<$ $i<n-k$.

\section{$2 \quad$ Algebraic auxiliary results}

This section is devoted to some algebraic results that are crucial for the proofs. Let $V$ and $W$ be finite dimensional real vector spaces equipped with non-degenerate inner products which, by abuse of notation, are both denoted by $\langle\cdot, \cdot\rangle$. The inner product of $V$ is assumed to be positive definite. We denote by $\operatorname{Hom}(V \times V, W)$ the space of all bilinear 
forms and by $\operatorname{Sym}(V \times V, W)$ its subspace that consists of all symmetric bilinear forms. The space $\operatorname{Sym}(V \times V, W)$ can be viewed as a complete metric space with respect to the usual Euclidean norm $\|\cdot\|$.

The Kulkarni-Nomizu product of two bilinear forms $\phi, \psi \in \operatorname{Hom}(V \times V, \mathbb{R})$ is the $(0,4)$-tensor $\phi \otimes \psi: V \times V \times V \times V \rightarrow \mathbb{R}$ defined by

$$
\begin{aligned}
\phi \otimes \psi\left(x_{1}, x_{2}, x_{3}, x_{4}\right)= & \phi\left(x_{1}, x_{3}\right) \psi\left(x_{2}, x_{4}\right)+\phi\left(x_{2}, x_{4}\right) \psi\left(x_{1}, x_{3}\right) \\
& -\phi\left(x_{1}, x_{4}\right) \psi\left(x_{2}, x_{3}\right)-\phi\left(x_{2}, x_{3}\right) \psi\left(x_{1}, x_{4}\right) .
\end{aligned}
$$

Using the inner product of $W$, we extend the Kulkarni-Nomizu product to bilinear forms $\beta, \gamma \in \operatorname{Hom}(V \times V, W)$, as the $(0,4)$-tensor $\beta \otimes \gamma: V \times V \times V \times V \rightarrow \mathbb{R}$ defined by

$$
\begin{aligned}
\beta \otimes \gamma\left(x_{1}, x_{2}, x_{3}, x_{4}\right)= & \left\langle\beta\left(x_{1}, x_{3}\right), \gamma\left(x_{2}, x_{4}\right)\right\rangle-\left\langle\beta\left(x_{1}, x_{4}\right), \gamma\left(x_{2}, x_{3}\right)\right\rangle \\
& +\left\langle\beta\left(x_{2}, x_{4}\right), \gamma\left(x_{1}, x_{3}\right)\right\rangle-\left\langle\beta\left(x_{2}, x_{3}\right), \gamma\left(x_{1}, x_{4}\right)\right\rangle .
\end{aligned}
$$

A bilinear form $\beta \in \operatorname{Hom}(V \times V, W)$ is called flat with respect to the inner product of $W$ if

$$
\left\langle\beta\left(x_{1}, x_{3}\right), \beta\left(x_{2}, x_{4}\right)\right\rangle-\left\langle\beta\left(x_{1}, x_{4}\right), \beta\left(x_{2}, x_{3}\right)\right\rangle=0
$$

for all $x_{1}, x_{2}, x_{3}, x_{4} \in V$, or equivalently if $\beta \otimes \beta=0$.

Associated to each bilinear form $\beta$ is the nullity space $\mathcal{N}(\beta)$ defined by

$$
\mathcal{N}(\beta)=\{x \in V: \beta(x, y)=0 \text { for all } y \in V\} .
$$

We need the following lemma, which was given in [30, Lemma 2.1].

Lemma 8. Let $\beta \in \operatorname{Sym}(V \times V, W)$ be a bilinear form, where $V$ and $W$ are both equipped with positive definite inner products and $\operatorname{dim} W \leq \operatorname{dim} V-2$. If $\beta \otimes \beta=\mu\langle\cdot, \cdot\rangle \otimes\langle\cdot, \cdot\rangle$ for some $\mu \neq 0$, then $\mu>0$ and there exist a unit vector $\xi \in W$ and a subspace $V_{1} \subseteq V$ such that

$$
\operatorname{dim} V_{1} \geq \operatorname{dim} V-\operatorname{dim} W+1
$$

and

$$
\beta(x, y)=\sqrt{\mu}\langle x, y\rangle \xi, \text { for all } x \in V_{1} \text { and } y \in V \text {. }
$$

We define the map scal: $\operatorname{Sym}(V \times V, W) \rightarrow \mathbb{R}$ by

$$
\operatorname{scal}(\beta)=\operatorname{trace} \operatorname{Ric}(\beta),
$$

where

$$
\operatorname{Ric}(\beta)(x, y)=\operatorname{trace} \mathrm{R}(\beta)(\cdot, x, \cdot, y), \quad x, y \in V \text { and } \mathrm{R}(\beta)=\frac{1}{2} \beta \otimes \beta .
$$

Hereafter, we assume that $V$ and $W$ are both endowed with positive definite inner products. For each $\beta \in \operatorname{Sym}(V \times V, W)$, we define the map

$$
\beta^{\sharp}: W \rightarrow \operatorname{End}(V), \quad \xi \mapsto \beta^{\sharp}(\xi)
$$


such that

$$
\left\langle\beta^{\sharp}(x), y\right\rangle=\langle\beta(x, y), \xi\rangle, \text { for all } x, y \in V,
$$

where $\operatorname{End}(V)$ denotes the set of all selfadjoint endomorphisms of $V$.

Let $\operatorname{dim} V=n$ and $\operatorname{dim} W=k$. When $2 \leq k \leq n / 2$, for each $\beta \in \operatorname{Sym}(V \times V, W)$, we denote by $\Phi(\beta)$ the subset of the unit $(k-1)$-sphere $\mathbb{S}^{k-1}$ in $W$ given by

$$
\Phi(\beta)=\left\{u \in \mathbb{S}^{k-1}: k \leq \operatorname{Index} \beta^{\sharp}(u) \leq n-k\right\} .
$$

The following proposition is crucial for the proof of Theorem 1.

Proposition 9. Given integers $2 \leq k \leq n / 2$ and $\lambda \in(1 / n, 1)$, there exists a positive constant $\varepsilon(n, k, \lambda)>0$, such that the following inequality holds

$\frac{1}{4}\left\|\beta \otimes \beta-\frac{\operatorname{scal}(\beta)}{n(n-1)}\langle\cdot, \cdot\rangle \otimes\langle\cdot, \cdot\rangle\right\|^{2}+\left(\|\beta\|^{2}-\lambda|\operatorname{trace} \beta|^{2}\right)_{+}^{2} \geq \varepsilon(n, k, \lambda)\left(\int_{\Lambda(\beta)}\left|\operatorname{det} \beta^{\sharp}(u)\right| d \mathbb{S}_{u}\right)^{4 / n}$

for any $\beta \in \operatorname{Sym}(V \times V, W)$, where

$$
\Lambda(\beta)= \begin{cases}\Phi(\beta), & \text { if } \operatorname{scal}(\beta)>0 \\ \mathbb{S}^{k-1}, & \text { if } \operatorname{scal}(\beta) \leq 0\end{cases}
$$

Proof: We consider the functions $\phi_{\lambda}, \psi: \operatorname{Sym}(V \times V, W) \rightarrow \mathbb{R}$ defined by

$$
\phi_{\lambda}(\beta)=\frac{1}{4}\left\|\beta \otimes \beta-\frac{\operatorname{scal}(\beta)}{n(n-1)}\langle\cdot, \cdot\rangle \otimes\langle\cdot, \cdot\rangle\right\|^{2}+\left(\|\beta\|^{2}-\lambda|\operatorname{trace} \beta|^{2}\right)_{+}^{2}
$$

and

$$
\psi(\beta)=\int_{\Lambda(\beta)}\left|\operatorname{det} \beta^{\sharp}(u)\right| d \mathbb{S}_{u} .
$$

We shall prove that $\phi_{\lambda}$ attains a positive minimum on $\Sigma_{n, k}$, where

$$
\Sigma_{n, k}=\{\beta \in \operatorname{Sym}(V \times V, W): \psi(\beta)=1\} .
$$

There exists a sequence $\left\{\beta_{m}\right\}$ in $\Sigma_{n, k}$ such that

$$
\lim _{m \rightarrow \infty} \phi_{\lambda}\left(\beta_{m}\right)=\inf \phi_{\lambda}\left(\Sigma_{n, k}\right) \geq 0
$$

We observe that $\beta_{m} \neq 0$ for all $m \in \mathbb{N}$, since $\beta_{m} \in \Sigma_{n, k}$. Then we may write $\beta_{m}=\left\|\beta_{m}\right\| \widehat{\beta}_{m}$, where $\left\|\widehat{\beta}_{m}\right\|=1$.

We claim that the sequence $\left\{\beta_{m}\right\}$ is bounded. Assume to the contrary that there exists a subsequence of $\left\{\beta_{m}\right\}$, which by abuse of notation is again denoted by $\left\{\beta_{m}\right\}$, such that $\lim _{m \rightarrow \infty}\left\|\beta_{m}\right\|=\infty$. Since $\left\|\widehat{\beta}_{m}\right\|=1$, we may assume, by taking a subsequence if necessary, that $\left\{\widehat{\beta}_{m}\right\}$ converges to some $\widehat{\beta} \in \operatorname{Sym}(V \times V, W)$ with $\|\widehat{\beta}\|=1$. Using 
the fact that $\phi_{\lambda}$ is homogeneous of degree 4 , we have $\phi_{\lambda}\left(\widehat{\beta}_{m}\right)=\phi_{\lambda}\left(\beta_{m}\right) /\left\|\beta_{m}\right\|^{4}$. Thus $\lim _{m \rightarrow \infty} \phi_{\lambda}\left(\widehat{\beta}_{m}\right)=0$ and consequently $\phi_{\lambda}(\widehat{\beta})=0$, or equivalently

$$
\widehat{\beta} \otimes \widehat{\beta}=\frac{\operatorname{scal}(\widehat{\beta})}{n(n-1)}\langle\cdot, \cdot\rangle \otimes\langle\cdot, \cdot\rangle
$$

and

$$
1 \leq \lambda|\operatorname{trace} \widehat{\beta}|^{2}=\lambda(\operatorname{scal}(\widehat{\beta})+1)
$$

Since $\lambda<1$, equation (3) implies scal $(\widehat{\beta})>0$. According to Lemma 8, there exists a unit vector $\widehat{\xi} \in W$ and subspace $\widehat{V}_{1}$ of $V$ with $\operatorname{dim} \widehat{V}_{1} \geq n-k+1$ such that

$$
\widehat{\beta}(x, y)=\left(\frac{\operatorname{scal}(\widehat{\beta})}{n(n-1)}\right)^{1 / 2}\langle x, y\rangle \widehat{\xi} \text { for all } x \in \widehat{V}_{1} \text { and } y \in V \text {. }
$$

Moreover, since $\left\{\beta_{m}\right\}$ is in $\Sigma_{n, k}$, there exists an open subset $\widehat{\mathcal{U}}_{m}$ of $\mathbb{S}^{k-1}$ such that $\widehat{\mathcal{U}}_{m} \subseteq$ $\Lambda\left(\widehat{\beta}_{m}\right)$ and $\operatorname{det} \widehat{\beta}_{m}^{\sharp}(u) \neq 0$ for all $u \in \widehat{\mathcal{U}}_{m}$ and $m \in \mathbb{N}$. From scal $(\widehat{\beta})>0$, we deduce that $\operatorname{scal}\left(\widehat{\beta}_{m}\right)>0$ and so $\widehat{\mathcal{U}}_{m} \subseteq \Phi\left(\widehat{\beta}_{m}\right)$ for $m$ large enough.

Let $\left\{\widehat{u}_{m}\right\}$ be a sequence such that $\widehat{u}_{m} \in \widehat{\mathcal{U}}_{m}$ for all $m \in \mathbb{N}$. We may assume that $\left\{\widehat{u}_{m}\right\}$ is convergent, by passing if necessary to a subsequence and set $\widehat{u}=\lim _{m \rightarrow \infty} \widehat{u}_{m}$. Since $\lim _{m \rightarrow \infty} \widehat{\beta}_{m}^{\sharp}\left(\widehat{u}_{m}\right)=\widehat{\beta}^{\sharp}(\widehat{u})$ and $\widehat{u}_{m} \in \widehat{\mathcal{U}}_{m}$, we deduce that Index $\widehat{\beta}^{\sharp}(\widehat{u}) \leq n-k$. Then, from (4) we obtain $\langle\widehat{\xi}, \widehat{u}\rangle \geq 0$. We claim that $\langle\widehat{\xi}, \widehat{u}\rangle=0$. Indeed, if $\langle\widehat{\xi}, \widehat{u}\rangle>0$, then (4) implies that $\widehat{\beta}^{\sharp}(\widehat{u})$ has at least $n-k+1$ positive eigenvalues and so, for $m$ large enough, $\widehat{\beta}_{m}^{\sharp}\left(\widehat{u}_{m}\right)$ has at least $n-k+1$ positive eigenvalues. This and the fact that $\operatorname{det} \widehat{\beta}_{m}^{\sharp}(u) \neq 0$ for all $u \in \widehat{\mathcal{U}}_{m}$, shows that $\widehat{\beta}_{m}^{\sharp}\left(\widehat{u}_{m}\right)$ has at most $k-1$ negative eigenvalues. Therefore, Index $\widehat{\beta}_{m}^{\sharp}\left(\widehat{u}_{m}\right) \leq k-1$ which is a contradiction, since $\widehat{u}_{m} \in \widehat{\mathcal{U}}_{m}$.

Thus, we have proved that for any convergent sequence $\left\{\widehat{u}_{m}\right\}$ such that $\widehat{u}_{m} \in \widehat{\mathcal{U}}_{m}$ for all $m$, we have $\left\langle\lim _{m \rightarrow \infty} \widehat{u}_{m}, \widehat{\xi}\right\rangle=0$.

Since $\widehat{\mathcal{U}}_{m}$ is open, we may choose convergent sequences $\left\{\widehat{u}_{m}^{(1)}\right\},\left\{\widehat{u}_{m}^{(2)}\right\}, \cdots,\left\{\widehat{u}_{m}^{(k)}\right\}$ in $\widehat{\mathcal{U}}_{m}$ such that $\widehat{u}_{m}^{(1)}, \widehat{u}_{m}^{(2)}, \cdots, \widehat{u}_{m}^{(k)}$ span $W$ for all $m \in \mathbb{N}$. From (4) and the fact that $\left\langle\lim _{m \rightarrow \infty} \widehat{u}_{m}^{(a)}, \widehat{\xi}\right\rangle=0$ for all $a \in\{1,2, \cdots, k\}$, we obtain that the restriction of $\widehat{\beta}_{m}$ to $\widehat{V}_{1} \times \widehat{V}_{1}$ satisfies

$$
\left.\lim _{m \rightarrow \infty} \widehat{\beta}_{m}\right|_{\widehat{V}_{1} \times \widehat{V}_{1}}=0
$$

and consequently

$$
\left.\lim _{m \rightarrow \infty}\left(\widehat{\beta}_{m} \otimes \widehat{\beta}_{m}\right)\right|_{\widehat{V}_{1} \times \widehat{V}_{1} \times \widehat{V}_{1} \times \widehat{V}_{1}}=0
$$

From the inequality

$$
\frac{1}{4}\left\|\left.\left(\widehat{\beta}_{m} \otimes \widehat{\beta}_{m}-\frac{\operatorname{scal}\left(\widehat{\beta}_{m}\right)}{n(n-1)}\langle\cdot, \cdot\rangle \otimes\langle\cdot, \cdot\rangle\right)\right|_{\widehat{V}_{1} \times \widehat{V}_{1} \times \widehat{V}_{1} \times \widehat{V}_{1}}\right\|^{2} \leq \phi_{\lambda}\left(\widehat{\beta}_{m}\right),
$$


(5) and the fact that $\lim _{m \rightarrow \infty} \phi_{\lambda}\left(\widehat{\beta}_{m}\right)=0$ we obtain scal $(\widehat{\beta})=0$, which contradicts $(3)$. Thus, the sequence $\left\{\beta_{m}\right\}$ is bounded, and it converges to some $\beta \in \operatorname{Sym}(V \times V, W)$, by taking a subsequence if necessary.

We claim that $\phi_{\lambda}(\beta)>0$. Arguing indirectly, we assume that $\phi_{\lambda}(\beta)=0$. Then

$$
\beta \otimes \beta=\frac{\operatorname{scal}(\beta)}{n(n-1)}\langle\cdot, \cdot\rangle \otimes\langle\cdot, \cdot\rangle
$$

and

$$
\|\beta\|^{2} \leq \lambda|\operatorname{trace} \beta|^{2}=\lambda\left(\operatorname{scal}(\beta)+\|\beta\|^{2}\right) .
$$

We notice that $\beta \neq 0$. Indeed, if $\beta=0$, then $\beta^{\sharp}(u)=0$ for all $u \in \mathbb{S}^{k-1}$. Since $\beta_{m} \in \Sigma_{n, k}$ for all $m \in \mathbb{N}$, there exists $\xi_{m} \in \Lambda\left(\beta_{m}\right)$ such that

$$
\left|\operatorname{det} \beta_{m}^{\sharp}\left(\xi_{m}\right)\right| \operatorname{Vol}\left(\Lambda\left(\beta_{m}\right)\right)=1 \text { for all } m \in \mathbb{N} \text {. }
$$

We may assume that $\xi_{m}$ converges to some $\xi$, by passing to a subsequence if necessary. Then $\lim _{m \rightarrow \infty} \beta_{m}^{\sharp}\left(\xi_{m}\right)=\beta^{\sharp}(\xi)=0$, which contradicts $(7)$. Therefore $\beta \neq 0$.

Now, from $(6)$ we obtain that $\operatorname{scal}(\beta) \neq 0$. Then, Lemma 8 implies that $\operatorname{scal}(\beta)>0$ and there exists a unit vector $\xi \in W$ and a subspace $V_{1}$ of $V$ with $\operatorname{dim} V_{1} \geq n-k+1$ such that

$$
\beta(x, y)=\left(\frac{\operatorname{scal}(\beta)}{n(n-1)}\right)^{1 / 2}\langle x, y\rangle \xi, \text { for all } x \in V_{1} \text { and } y \in V .
$$

Since $\beta_{m} \in \Sigma_{n, k}$ for all $m \in \mathbb{N}$, there exists an open subset $\mathcal{U}_{m}$ of $\mathbb{S}^{k-1}$ such that $\mathcal{U}_{m} \subseteq \Lambda\left(\beta_{m}\right)$ and $\operatorname{det} \beta_{m}^{\sharp}(u) \neq 0$, for all $u \in \mathcal{U}_{m}$ and $m \in \mathbb{N}$. Moreover, we have that $\operatorname{scal}\left(\beta_{m}\right)>0$ and so $\mathcal{U}_{m} \subseteq \Phi\left(\beta_{m}\right)$ for $m$ large enough.

Let $\left\{u_{m}\right\}$ be a sequence such that $u_{m} \in \mathcal{U}_{m}$ for all $m \in \mathbb{N}$. We may assume that $u_{m}$ is convergent, by passing if necessary to a subsequence and set $u=\lim _{m \rightarrow \infty} u_{m}$. Since $\lim _{m \rightarrow \infty} \beta_{m}^{\sharp}\left(u_{m}\right)=\beta^{\sharp}(u)$ and $u_{m} \in \mathcal{U}_{m}$ it follows that Index $\beta^{\sharp}(u) \leq n-k$. Then, from (8) we get $\langle\xi, u\rangle \geq 0$. We claim that $\langle\xi, u\rangle=0$. Indeed, if $\langle\xi, u\rangle>0$ then (8) implies that $\beta^{\sharp}(u)$ has at least $n-k+1$ positive eigenvalues and so, for $m$ large enough, $\beta_{m}^{\sharp}\left(u_{m}\right)$ has at least $n-k+1$ positive eigenvalues. This and the fact that $\operatorname{det} \beta_{m}^{\sharp}(u) \neq 0$ for all $u \in \mathcal{U}_{m}$ and $m \in \mathbb{N}$, shows that $\beta_{m}^{\sharp}\left(u_{m}\right)$ has at most $k-1$ negative eigenvalues, which is a contradiction, since $u_{m} \in \mathcal{U}_{m}$ for all $m \in \mathbb{N}$.

Thus, we have proved that for any convergent sequence $\left\{u_{m}\right\}$ such that $u_{m} \in \mathcal{U}_{m}$ for all $m$, we have $\left\langle\lim _{m \rightarrow \infty} u_{m}, \xi\right\rangle=0$. Since $\mathcal{U}_{m}$ is open, we may choose convergent sequences $\left\{u_{m}^{(1)}\right\},\left\{u_{m}^{(2)}\right\}, \cdots,\left\{u_{m}^{(k)}\right\}$ in $\mathcal{U}_{m}$ such that $u_{m}^{(1)}, u_{m}^{(2)}, \cdots, u_{m}^{(k)}$ span $W$ for all $m \in \mathbb{N}$. Then, from (8) and the fact that $\left\langle\lim _{m \rightarrow \infty} u_{m}^{(a)}, \xi\right\rangle=0$ for all $a \in\{1,2, \cdots, k\}$ we obtain that the restriction of $\beta_{m}$ to $V_{1} \times V_{1}$ satisfies

$$
\left.\lim _{m \rightarrow \infty} \beta_{m}\right|_{V_{1} \times V_{1}}=0
$$

and consequently

$$
\left.\lim _{m \rightarrow \infty}\left(\beta_{m} \otimes \beta_{m}\right)\right|_{V_{1} \times V_{1} \times V_{1} \times V_{1}}=0
$$


From the inequality

$$
\frac{1}{4}\left\|\left.\left(\beta_{m} \otimes \beta_{m}-\frac{\operatorname{scal}\left(\beta_{m}\right)}{n(n-1)}\langle\cdot, \cdot\rangle \otimes\langle\cdot, \cdot\rangle\right)\right|_{V_{1} \times V_{1} \times V_{1} \times V_{1}}\right\|^{2} \leq \phi_{\lambda}\left(\beta_{m}\right),
$$

(9) and the fact that $\lim _{m \rightarrow \infty} \phi_{\lambda}\left(\beta_{m}\right)=0$ we obtain $\operatorname{scal}(\beta)=0$, which contradicts $(6)$. Thus, we have proved that $\phi_{\lambda}(\beta)>0$ and so $\phi_{\lambda}$ attains a positive minimum on $\Sigma_{n, k}$ which obviously depends only on $n, k$ and $\lambda$ and is denoted by $\varepsilon(n, k, \lambda)$.

Now, let $\beta \in \operatorname{Sym}(V \times V, W)$. Assume that $\psi(\beta) \neq 0$ and set $\gamma=\beta /(\psi(\beta))^{1 / n}$. Clearly $\gamma \in \Sigma_{n, k}$, and consequently $\phi_{\lambda}(\gamma) \geq \varepsilon(n, k, \lambda)$. Since $\phi_{\lambda}$ is homogeneous of degree 4 , the desired inequality is obviously fulfilled. In the case where $\psi(\beta)=0$, the inequality is trivial.

We also need the following result on flat bilinear forms, which is due to Moore [19, Proposition 2].

Lemma 10. Let $\beta \in \operatorname{Sym}(V \times V, U)$ be a flat bilinear form with respect to a Lorentzian inner product of $U$. If $\operatorname{dim} V>\operatorname{dim} U$ and $\beta(x, x) \neq 0$ for all non-zero $x \in V$, then there is a non-zero isotropic vector $e \in U$ and a bilinear form $\phi \in \operatorname{Sym}(V \times V, \mathbb{R})$ such that $\operatorname{dim} \mathcal{N}(\beta-e \phi) \geq \operatorname{dim} V-\operatorname{dim} U+2$.

We define the map W: $\operatorname{Sym}(V \times V, W) \rightarrow \operatorname{Hom}(V \times V \times V \times V, \mathbb{R})$ by

$$
\mathrm{W}(\beta)=\mathrm{R}(\beta)-\mathrm{L}(\beta) \otimes\langle\cdot, \cdot\rangle,
$$

where

$$
\mathrm{L}(\beta)=\frac{1}{n-2}\left(\operatorname{Ric}(\beta)-\frac{\operatorname{scal}(\beta)}{2(n-1)}\langle\cdot, \cdot\rangle\right),
$$

The following lemma is in fact contained in [19]. For the sake of completeness we give a short proof.

Lemma 11. Let $\beta \in \operatorname{Sym}(V \times V, W)$ be a bilinear form and $\operatorname{dim} W<\operatorname{dim} V-2$. If $\mathrm{W}(\beta)=0$, then there exists a vector $\xi \in W$ and a subspace $V_{1} \subseteq V$ such that

$$
\operatorname{dim} V_{1} \geq \operatorname{dim} V-\operatorname{dim} W
$$

and

$$
\beta(x, y)=\langle x, y\rangle \xi, \text { for all } x \in V_{1} \text { and } y \in V \text {. }
$$

Proof: We endow the vector space $\widetilde{W}=W \oplus \mathbb{R}^{2}$ with the Lorentzian inner product $\langle\langle\cdot, \cdot\rangle\rangle$ given by

$$
\left\langle\left\langle\left(\xi,\left(s_{1}, s_{2}\right)\right),\left(\eta,\left(t_{1}, t_{2}\right)\right)\right\rangle\right\rangle=\langle\xi, \eta\rangle+s_{1} t_{2}+s_{2} t_{1}
$$

and define the symmetric bilinear form $\widetilde{\beta}: V \times V \rightarrow \widetilde{W}$ by

$$
\widetilde{\beta}(x, y)=(\beta(x, y),\langle x, y\rangle,-\mathrm{L}(\beta)(x, y)) .
$$


Since $\mathbf{W}(\beta)=0$ it follows that $\widetilde{\beta}$ is flat with respect to $\langle\langle\cdot, \cdot\rangle\rangle$. From Lemma 10, we know that there exists a non-zero isotropic vector $e=(\eta,(s, t)) \in \widetilde{W}$ and a symmetric bilinear form $\phi: V \times V \rightarrow \mathbb{R}$ such that $\operatorname{dim} \mathcal{N}(\widetilde{\beta}-\phi e) \geq \operatorname{dim} V-\operatorname{dim} W$. By setting $V_{1}=\mathcal{N}(\widetilde{\beta}-e \phi)$, we have that $\widetilde{\beta}(x, y)=\phi(x, y) e$, or equivalently

$$
\beta(x, y)=\phi(x, y) \eta, \quad\langle x, y\rangle=s \phi(x, y) \text { and } \mathrm{L}(\beta)(x, y)=-t \phi(x, y),
$$

for all $x \in V_{1}$ and $y \in V$. Therefore, $\beta(x, y)=\langle x, y\rangle \xi$, where $\xi=(1 / s) \eta$.

When $2 \leq k \leq[(n-2) / 2]$, then for each $\beta \in \operatorname{Sym}(V \times V, W)$, we define the subset $\Omega(\beta)$ of the unit $(k-1)$-sphere $\mathbb{S}^{k-1}$ in $W$ given by

$$
\Omega(\beta)=\left\{u \in \mathbb{S}^{k-1}: k<\operatorname{Index} \beta^{\sharp}(u)<n-k\right\} .
$$

The following proposition is crucial for the proof of Theorem 6 .

Proposition 12. Given integers $2 \leq k \leq[(n-2) / 2]$ and $\lambda \in(1 / n, 1)$, there exists a positive constant $\varepsilon_{1}(n, k, \lambda)$ such that the following inequality holds

$$
\|\mathrm{W}(\beta)\|^{2}+\left(\|\beta\|^{2}-\lambda|\operatorname{trace} \beta|^{2}\right)_{+}^{2} \geq \varepsilon_{1}(n, k, \lambda)\left(\int_{\Omega(\beta)}\left|\operatorname{det} \beta^{\sharp}(u)\right| d \mathbb{S}_{u}\right)^{4 / n},
$$

for all $\beta \in \operatorname{Sym}(V \times V, W)$.

Proof: We consider the functions $\phi_{\lambda}, \psi: \operatorname{Sym}(V \times V, W) \rightarrow \mathbb{R}$ defined by

$$
\phi_{\lambda}(\beta)=\|\mathbf{W}(\beta)\|^{2}+\left(\|\beta\|^{2}-\lambda|\operatorname{trace} \beta|^{2}\right)_{+}^{2} \quad \text { and } \quad \psi(\beta)=\int_{\Omega(\beta)}\left|\operatorname{det} \beta^{\sharp}(u)\right| d \mathbb{S}_{u} .
$$

In order to prove the desired inequality, it is sufficient to show that $\phi_{\lambda}$ attaints a positive minimum on $\Sigma_{n, k}$, where

$$
\Sigma_{n, k}=\{\beta \in \operatorname{Sym}(V \times V, W): \psi(\beta)=1\} .
$$

There exists a sequence $\left\{\beta_{m}\right\}$ in $\Sigma_{n, k}$ such that

$$
\lim _{m \rightarrow \infty} \phi_{\lambda}\left(\beta_{m}\right)=\inf \phi_{\lambda}\left(\Sigma_{n, k}\right) \geq 0
$$

We observe that $\beta_{m} \neq 0$ for all $m \in \mathbb{N}$, since $\beta_{m} \in \Sigma_{n, k}$. Thus, we may write $\beta_{m}=$ $\left\|\beta_{m}\right\| \widehat{\beta}_{m}$, where $\left\|\widehat{\beta}_{m}\right\|=1$.

We claim that the sequence $\left\{\beta_{m}\right\}$ is bounded. Assume to the contrary that there exists a subsequence of $\left\{\beta_{m}\right\}$, which by abuse of notation is again denoted by $\left\{\beta_{m}\right\}$, such that $\lim _{m \rightarrow \infty}\left\|\beta_{m}\right\|=\infty$. Since $\left\|\widehat{\beta}_{m}\right\|=1$, we may assume, by taking a subsequence if necessary, that $\left\{\widehat{\beta}_{m}\right\}$ converges to some $\widehat{\beta} \in \operatorname{Sym}(V \times V, W)$ with $\|\widehat{\beta}\|=1$. Using 
the fact that $\phi_{\lambda}$ is homogeneous of degree 4 , we obtain $\phi_{\lambda}\left(\widehat{\beta}_{m}\right)=\phi_{\lambda}\left(\beta_{m}\right) /\left\|\beta_{m}\right\|^{4}$. Thus $\lim _{m \rightarrow \infty} \phi_{\lambda}\left(\widehat{\beta}_{m}\right)=0$ and consequently $\phi_{\lambda}(\widehat{\beta})=0$, or equivalently $\mathrm{W}(\widehat{\beta})=0$ and

$$
1 \leq \lambda|\operatorname{trace} \widehat{\beta}|^{2}=\lambda(\operatorname{scal}(\widehat{\beta})+1)
$$

According to Lemma 11, we have that there exist a vector subspace $\widehat{V}_{1}$ of $V$ with $\operatorname{dim} \widehat{V}_{1} \geq$ $n-k$ and a vector $\widehat{\xi} \in W$ such that

$$
\widehat{\beta}(x, y)=\langle x, y\rangle \widehat{\xi} \text { for all } x \in \widehat{V}_{1} \text { and } y \in V .
$$

Moreover, since $\beta_{m} \in \Sigma_{n, k}$ there exists an open subset $\widehat{\mathcal{U}}_{m}$ of $\mathbb{S}^{k-1}$ such that $\widehat{\mathcal{U}}_{m} \subseteq \Omega\left(\widehat{\beta}_{m}\right)$ and $\operatorname{det} \widehat{\beta}_{m}^{\sharp}(u) \neq 0$ for all $u \in \widehat{\mathcal{U}}_{m}$ and $m \in \mathbb{N}$.

Let $\left\{\widehat{u}_{m}\right\}$ be a sequence such that $\widehat{u}_{m} \in \widehat{\mathcal{U}}_{m}$ for all $m \in \mathbb{N}$. We may assume that $\left\{\widehat{u}_{m}\right\}$ is convergent, by passing if necessary to a subsequence and set $\widehat{u}=\lim _{m \rightarrow \infty} \widehat{u}_{m}$. Since $\lim _{m \rightarrow \infty} \widehat{\beta}_{m}^{\sharp}\left(\widehat{u}_{m}\right)=\widehat{\beta}^{\sharp}(\widehat{u})$ and $\widehat{u}_{m} \in \widehat{\mathcal{U}}_{m}$ it follows that Index $\widehat{\beta}^{\sharp}(\widehat{u})<n-k$. Then, from (12) we get $\langle\widehat{\xi}, \widehat{u}\rangle \geq 0$. We claim that $\langle\widehat{\xi}, \widehat{u}\rangle=0$. Indeed, if $\langle\widehat{\xi}, \widehat{u}\rangle>0$ then (12) implies that $\widehat{\beta}^{\sharp}(\widehat{u})$ has at least $n-k$ positive eigenvalues and so, for $m$ large enough $\widehat{\beta}_{m}^{\sharp}\left(\widehat{u}_{m}\right)$ has at least $n-k$ positive eigenvalues. On account of the fact that $\operatorname{det} \widehat{\beta}_{m}^{\sharp}(u) \neq 0$ for all $u \in \widehat{\mathcal{U}}_{m}$ and $m \in \mathbb{N}$, we have that $\widehat{\beta}_{m}^{\sharp}\left(\widehat{u}_{m}\right)$ has at most $k$ negative eigenvalues, which is a contradiction, since $\widehat{u}_{m} \in \widehat{\mathcal{U}}_{m}$ for all $m \in \mathbb{N}$.

Thus, we have proved that for any convergent sequence $\left\{\widehat{u}_{m}\right\}$ such that $\widehat{u}_{m} \in \widehat{\mathcal{U}}_{m}$ for all $m \in \mathbb{N}$, we have $\left\langle\lim _{m \rightarrow \infty} \widehat{u}_{m}, \widehat{\xi}\right\rangle=0$. Since $\widehat{\mathcal{U}}_{m}$ is open, we may choose convergent sequences $\left\{\widehat{u}_{m}^{(1)}\right\},\left\{\widehat{u}_{m}^{(2)}\right\}, \cdots,\left\{\widehat{u}_{m}^{(k)}\right\}$ in $\widehat{\mathcal{U}}_{m}$ such that $\widehat{u}_{m}^{(1)}, \widehat{u}_{m}^{(2)}, \cdots, \widehat{u}_{m}^{(k)}$ span $W$ for all $m \in$ $\mathbb{N}$. Then, from $(12)$ and the fact that $\left\langle\lim _{m \rightarrow \infty} \widehat{u}_{m}^{(a)}, \widehat{\xi}\right\rangle=0$ for all $a \in\{1,2, \cdots, k\}$ we have that the restriction of $\widehat{\beta}_{m}$ to $\widehat{V}_{1} \times \widehat{V}_{1}$ satisfies

$$
\left.\lim _{m \rightarrow \infty} \widehat{\beta}_{m}\right|_{\widehat{V}_{1} \times \widehat{V}_{1}}=0
$$

and consequently

$$
\left.\lim _{m \rightarrow \infty} \mathrm{R}\left(\widehat{\beta}_{m}\right)\right|_{\widehat{V}_{1} \times \widehat{V}_{1} \times \widehat{V}_{1} \times \widehat{V}_{1}}=0 \text { and }\left.\lim _{m \rightarrow \infty} \mathrm{L}\left(\widehat{\beta}_{m}\right)\right|_{\widehat{V}_{1} \times \widehat{V}_{1}}=-\left.\frac{\operatorname{scal}(\widehat{\beta})}{2(n-1)(n-2)}\langle\cdot, \cdot\rangle\right|_{\widehat{V}_{1} \times \widehat{V}_{1}} .
$$

From the inequality

$$
\left\|\left.\mathrm{W}\left(\widehat{\beta}_{m}\right)\right|_{\widehat{V}_{1} \times \widehat{V}_{1} \times \widehat{V}_{1} \times \widehat{V}_{1}}\right\|^{2} \leq \phi_{\lambda}\left(\widehat{\beta}_{m}\right),
$$

(13) and the fact that $\lim _{m \rightarrow \infty} \phi_{\lambda}\left(\widehat{\beta}_{m}\right)=0$ we obtain scal $(\widehat{\beta})=0$, which contradicts (11). Thus, the sequence $\left\{\beta_{m}\right\}$ is bounded and converges to some $\beta \in \operatorname{Sym}(V \times V, W)$, by taking a subsequence if necessary.

We claim that $\phi_{\lambda}(\beta)>0$. Arguing indirectly, we assume that $\phi_{\lambda}(\beta)=0$. Then, we have $\mathbf{W}(\beta)=0$ and

$$
\|\beta\|^{2} \leq \lambda|\operatorname{trace} \beta|^{2}=\lambda\left(\operatorname{scal}(\beta)+\|\beta\|^{2}\right) .
$$


According to Lemma 11, there exist a vector subspace $V_{1}$ of $V$ with $\operatorname{dim} V_{1} \geq n-k$ and a vector $\xi \in W$ such that

$$
\beta(x, y)=\langle x, y\rangle \xi \text { for all } x \in V_{1} \text { and } y \in V .
$$

We notice that $\beta \neq 0$. Indeed, if $\beta=0$, then $\beta^{\sharp}(u)=0$ for all $u \in \mathbb{S}^{k-1}$. Since $\beta_{m} \in \Sigma_{n, k}$ for all $m \in \mathbb{N}$ there exists $\xi_{m} \in \Omega\left(\beta_{m}\right)$ such that

$$
\left|\operatorname{det} \beta_{m}^{\sharp}\left(\xi_{m}\right)\right| \operatorname{Vol}\left(\Omega\left(\beta_{m}\right)\right)=1 \text { for all } m \in \mathbb{N} \text {. }
$$

We may assume that the sequence $\left\{\xi_{m}\right\}$ converges to some $\xi \in \mathbb{S}^{k-1}$, by passing again to a subsequence if necessary. Then $\lim _{m \rightarrow \infty} \beta_{m}^{\sharp}\left(\xi_{m}\right)=\beta^{\sharp}(\xi)=0$, which contradicts (16). Therefore $\beta \neq 0$.

From the fact that $\beta_{m} \in \Sigma_{n, k}$ for all $m \in \mathbb{N}$, we deduce that there exists an open subset $\mathcal{U}_{m}$ of $\mathbb{S}^{k-1}$ such that $\mathcal{U}_{m} \subseteq \Omega\left(\beta_{m}\right)$ and $\operatorname{det} \beta_{m}^{\sharp}(u) \neq 0$ for all $u \in \mathcal{U}_{m}$ and $m \in \mathbb{N}$. Let $\left\{u_{m}\right\}$ be a sequence such that $u_{m} \in \mathcal{U}_{m}$ for all $m \in \mathbb{N}$. We may assume that $u_{m}$ is convergent, by passing if necessary to a subsequence, and set $u=\lim _{m \rightarrow \infty} u_{m}$. Since $\lim _{m \rightarrow \infty} \beta_{m}^{\sharp}\left(u_{m}\right)=\beta^{\sharp}(u)$ and $u_{m} \in \mathcal{U}_{m}$, it follows that Index $\beta^{\sharp}(u)<n-k$. Then, from (15) we get $\langle\xi, u\rangle \geq 0$. We claim that $\langle\xi, u\rangle=0$. Indeed, if $\langle\xi, u\rangle>0$, then (15) implies that $\beta^{\sharp}(u)$ has at least $n-k$ positive eigenvalues and so, for $m$ large enough, $\beta_{m}^{\sharp}\left(u_{m}\right)$ has at least $n-k$ positive eigenvalues. This and the fact that $\operatorname{det} \beta_{m}^{\sharp}(u) \neq 0$ for all $u \in \mathcal{U}_{m}$ and $m \in \mathbb{N}$, shows that $\beta_{m}^{\sharp}\left(u_{m}\right)$ has at most $k$ negative eigenvalues, which is a contradiction, since $u_{m} \in \mathcal{U}_{m}$ for all $m \in \mathbb{N}$.

Thus, we have proved that for any convergent sequence $\left\{u_{m}\right\}$ such that $u_{m} \in \mathcal{U}_{m}$ for all $m \in \mathbb{N}$, we have $\left\langle\lim _{m \rightarrow \infty} u_{m}, \xi\right\rangle=0$. Since $\mathcal{U}_{m}$ is open, we may choose convergent sequences $\left\{u_{m}^{(1)}\right\},\left\{u_{m}^{(2)}\right\}, \cdots,\left\{u_{m}^{(k)}\right\}$ in $\mathcal{U}_{m}$ such that $u_{m}^{(1)}, u_{m}^{(2)}, \cdots, u_{m}^{(k)}$ span $W$ for all $m \in$ $\mathbb{N}$. From $(15)$ and the fact that $\left\langle\lim _{m \rightarrow \infty} u_{m}^{(a)}, \xi\right\rangle=0$ for all $a \in\{1,2, \cdots, k\}$, we obtain that the restriction of $\beta_{m}$ to $V_{1} \times V_{1}$ satisfies

$$
\left.\lim _{m \rightarrow \infty} \beta_{m}\right|_{V_{1} \times V_{1}}=0
$$

and consequently

$$
\left.\lim _{m \rightarrow \infty} \mathrm{R}\left(\beta_{m}\right)\right|_{V_{1} \times V_{1} \times V_{1} \times V_{1}}=0 \text { and }\left.\lim _{m \rightarrow \infty} \mathrm{L}\left(\beta_{m}\right)\right|_{V_{1} \times V_{1}}=-\left.\frac{\operatorname{scal}(\beta)}{2(n-1)(n-2)}\langle\cdot, \cdot\rangle\right|_{V_{1} \times V_{1}} .
$$

From the inequality

$$
\left\|\left.\mathrm{W}\left(\beta_{m}\right)\right|_{V_{1} \times V_{1} \times V_{1} \times V_{1}}\right\|^{2} \leq \phi_{\lambda}\left(\beta_{m}\right),
$$

(17) and the fact that $\lim _{m \rightarrow \infty} \phi_{\lambda}\left(\beta_{m}\right)=0$ we obtain $\operatorname{scal}(\beta)=0$, which contradicts (14). Thus, we have proved that $\phi_{\lambda}(\beta)>0$ and so $\phi_{\lambda}$ attains a positive minimum on $\Sigma_{n, k}$ which obviously depends only on $n, k$ and $\lambda$ and is denoted by $\varepsilon_{1}(n, k, \lambda)$.

Now, let $\beta \in \operatorname{Sym}(V \times V, W)$. Assume that $\psi(\beta) \neq 0$ and set $\gamma=\beta /(\psi(\beta))^{1 / n}$. Clearly $\gamma \in \Sigma_{n, k}$, and consequently $\phi_{\lambda}(\gamma) \geq \varepsilon_{1}(n, k, \lambda)$. Since $\phi_{\lambda}$ is homogeneous of degree 4 , the desired inequality is obviously fulfilled. In the case where $\psi(\beta)=0$, the inequality is trivial. 
Remark 13. In the case where $\lambda \leq 1 / n$, arguing as in the proof of Proposition 9 , we have that there exist a positive constant $d(n, k)$ such that

$$
\left(\|\beta\|^{2}-\lambda|\operatorname{trace} \beta|^{2}\right)^{2} \geq\left(\|\beta\|^{2}-\frac{1}{n}|\operatorname{trace} \beta|^{2}\right)^{2} \geq d(n, k)\left(\int_{\mathbb{S}^{k-1}}\left|\operatorname{det} \beta^{\sharp}(u)\right| d \mathbb{S}_{u}\right)^{4 / n}
$$

for any $\beta \in \operatorname{Sym}(V \times V, W)$.

However, if $\lambda \in(1 / n, 1)$ then the first term of the LHS of inequalities (2) and (10) is essential and cannot be dropped. For instance, let $n \geq 7, k=2$ and $\left\{\xi_{1}, \xi_{2}\right\}$ be an orthonormal basis of $W$. Consider $\beta \in \operatorname{Sym}(V \times V, W)$ defined by $\beta(x, y)=\langle A x, y\rangle \xi_{1}$, where $A=\operatorname{diag}(a, a,-a,-a, \cdots,-a), a>0$. For any $\lambda \in\left[n /(n-4)^{2}, 1\right)$ we have that $\left(\|\beta\|^{2}-\lambda|\operatorname{trace} \beta|^{2}\right)_{+}=0$. Moreover, $\Phi(\beta)=\mathbb{S}^{1} \backslash\left\{ \pm \xi_{2}\right\}$ and this shows that inequality (2) cannot hold by dropping the first term of the LHS.

\section{The proofs}

We recall some well known facts on the total curvature and how Morse theory provides restrictions on the Betti numbers. Let $f:\left(M^{n}, g\right) \rightarrow \mathbb{R}^{n+k}$ be an isometric immersion of a compact, connected and oriented $n$-dimensional Riemannian manifold into the $(n+k)$ dimensional Euclidean space $\mathbb{R}^{n+k}$ equipped with the usual inner product $\langle\cdot, \cdot\rangle$. The normal bundle of $f$ is given by

$$
N_{f} M=\left\{(p, \xi) \in f^{*}\left(T \mathbb{R}^{n+k}\right): \xi \perp d f_{p}\left(T_{p} M\right)\right\}
$$

and the corresponding unit normal bundle is defined by

$$
U N_{f}=\left\{(p, \xi) \in N_{f} M:|\xi|=1\right\}
$$

where $f^{*}\left(T \mathbb{R}^{n+k}\right)$ is the induced bundle of $f$.

The generalized Gauss map $\nu: U N_{f} \rightarrow \mathbb{S}^{n+k-1}$ is defined by $\nu(p, \xi)=\xi$, where $\mathbb{S}^{n+k-1}$ is the unit $(n+k-1)$-dimensional sphere of $\mathbb{R}^{n+k}$. For each $u \in \mathbb{S}^{n+k-1}$, we consider the height function $h_{u}: M^{n} \rightarrow \mathbb{R}$ defined by $h_{u}(p)=\langle f(p), u\rangle, p \in M^{n}$. Since $h_{u}$ has a degenerate critical point if and only if $u$ is a critical point of the generalized Gauss map, by Sard's theorem there exists a subset $E \subset \mathbb{S}^{n+k-1}$ of measure zero such that $h_{u}$ is a Morse function for all $u \in \mathbb{S}^{n+k-1} \backslash E$. For each $u \in \mathbb{S}^{n+k-1} \backslash E$, we denote by $\mu_{i}(u)$ the number of critical points of $h_{u}$ of index $i$. We also set $\mu_{i}(u)=0$ for any $u \in E$. Following Kuiper [14], we define the total curvature of index $i$ of $f$ by

$$
\tau_{i}(f)=\frac{1}{\operatorname{Vol}\left(\mathbb{S}^{n+k-1}\right)} \int_{\mathbb{S}^{n+k-1}} \mu_{i}(u) d \mathbb{S},
$$

where $d \mathbb{S}$ denotes the volume element of the sphere $\mathbb{S}^{n+k-1}$. 
Let $\beta_{i}\left(M^{n} ; \mathbb{F}\right)=\operatorname{dim}_{\mathbb{F}} H_{i}\left(M^{n} ; \mathbb{F}\right)$ be the $i$-th Betti number of $M^{n}$ over an arbitrary coefficient field $\mathbb{F}$. From the weak Morse inequalities (cf. [17]) we have $\mu_{i}(u) \geq \beta_{i}\left(M^{n} ; \mathbb{F}\right)$, for all $u \in \mathbb{S}^{n+k-1} \backslash E$. By integrating over $\mathbb{S}^{n+k-1}$, we obtain

$$
\tau_{i}(f) \geq \beta_{i}\left(M^{n} ; \mathbb{F}\right) .
$$

For each $(p, \xi) \in U N_{f}$, we denote by $A_{\xi}$ the shape operator of $f$ in the direction $\xi$ which is given by

$$
g\left(A_{\xi} X, Y\right)=\langle\alpha(X, Y), \xi\rangle, \quad X, Y \in T M,
$$

where $\alpha$ is the second fundamental form of $f$ viewed as a section of the vector bundle $\operatorname{Hom}\left(T M \times T M, N_{f} M\right)$. There is a natural volume element $d \Sigma$ on the unit normal bundle $U N_{f}$. In fact, if $d V$ is a $(k-1)$-form on $U N_{f}$ such that its restriction to a fiber of the unit normal bundle at $(p, \xi)$ is the volume element of the unit $(k-1)$-sphere of the normal space of $f$ at $p$, then $d \Sigma=d M \wedge d V$, where $d M$ is the volume element of $M^{n}$ with respect to the metric $g$. Furthermore, we have

$$
\nu^{*}(d \mathbb{S})=G(p, \xi) d \Sigma,
$$

where $G(p, \xi)=(-1)^{n} \operatorname{det} A_{\xi}$ is the Lipschitz-Killing curvature at $(p, \xi) \in U N_{f}$.

The total absolute curvature $\tau(f)$ of $f$ in the sense of Chern and Lashof is defined by

$$
\tau(f)=\frac{1}{\operatorname{Vol}\left(\mathbb{S}^{n+k-1}\right)} \int_{U N_{f}}\left|\nu^{*}(d \mathbb{S})\right|=\frac{1}{\operatorname{Vol}\left(\mathbb{S}^{n+k-1}\right)} \int_{U N_{f}}\left|\operatorname{det} A_{\xi}\right| d \Sigma .
$$

We need the following result which is due to Chern and Lashof $[11,12]$.

Theorem 14. Let $f: M^{n} \rightarrow \mathbb{R}^{n+k}$ be an isometric immersion of a compact, connected and oriented $n$-dimensional Riemannian manifold $M^{n}$ into $\mathbb{R}^{n+k}$. Then the total absolute curvature of $f$ satisfies the inequality

$$
\tau(f) \geq \sum_{i=0}^{n} \beta_{i}\left(M^{n} ; \mathbb{F}\right)
$$

Shiohama and $\mathrm{Xu}[25$, p. 381] proved that

$$
\int_{U^{i} N_{f}}\left|\operatorname{det} A_{\xi}\right| d \Sigma=\int_{\mathbb{S}^{n+k-1}} \mu_{i}(u) d \mathbb{S}
$$

where $U^{i} N_{f}$, is the subset of the unit normal bundle of $f$ defined by

$$
U^{i} N_{f}=\left\{(p, \xi) \in U N_{f}: \text { Index } A_{\xi}=i\right\}, \quad 0 \leq i \leq n .
$$

The $(0,4)$-Riemann curvature tensor $R$ of $M^{n}$ is related to the second fundamental form of $f$ via the Gauss equation

$$
R(X, Y, Z, W)=\langle\alpha(X, Z), \alpha(Y, W)\rangle-\langle\alpha(X, W), \alpha(Y, Z)\rangle, \quad X, Y, Z, W \in T M .
$$


In terms of the Kulkarni-Nomizu product, the Gauss equation is written equivalently as

$$
R=\frac{1}{2} \alpha \oplus \alpha
$$

On the other hand, $R$ decomposes as

$$
R=\mathcal{W}+\mathcal{L} \otimes g
$$

where $\mathcal{W}$ is the Weyl tensor and

$$
\mathcal{L}=\frac{1}{n-2}\left(\text { Ric }-\frac{\text { scal }}{2(n-1)} g\right) .
$$

is the Schouten tensor of $M^{n}$.

We are now able to present the proofs of our results.

Proof of Theorem 1: Let $f: M^{n} \rightarrow \mathbb{R}^{n+k}$ be an isometric immersion with second fundamental form $\alpha$ and shape operator $A_{\xi}$ with respect to $\xi$, where $(p, \xi) \in U N_{f}$. Using the Gauss equation and Proposition 9 we have

$$
\left(\left\|R-\frac{\text { scal }}{n(n-1)} R_{1}\right\|^{2}+\left(S-\delta n^{2} H^{2}\right)_{+}^{2}\right)^{n / 4}(p) \geq(\varepsilon(n, k, \delta))^{n / 4} \int_{\Lambda(\alpha(p))}\left|\operatorname{det} A_{\xi}\right| d V_{\xi}
$$

for all $p \in M^{n}$. Integrating over $M^{n}$ and using (19), we obtain

$$
\int_{M^{n}}\left(\left\|R-\frac{\text { scal }}{n(n-1)} R_{1}\right\|^{2}+\left(S-\delta n^{2} H^{2}\right)_{+}^{2}\right)^{n / 4} d M \geq(\varepsilon(n, k, \delta))^{n / 4} \operatorname{Vol}\left(\mathbb{S}^{n+k-1}\right) \sum_{i=k}^{n-k} \tau_{i}(f) .
$$

Observe that

$$
\begin{gathered}
\left(\left\|R-\frac{\text { scal }}{n(n-1)} R_{1}\right\|^{2}+\left(S-\delta n^{2} H^{2}\right)_{+}^{2}\right)^{n / 4}(p) \leq \\
2^{(n-4) / 4}\left(\left\|R-\frac{\text { scal }}{n(n-1)} R_{1}\right\|^{n / 2}+\left(S-\delta n^{2} H^{2}\right)_{+}^{n / 2}\right)(p)
\end{gathered}
$$

for all $p \in M^{n}$. Thus, from (20) and (18) we obtain

$$
\begin{array}{rl}
\int_{M^{n}}\left\|R-\frac{\mathrm{scal}}{n(n-1)} R_{1}\right\|^{n / 2} & d M+\int_{M^{n}}\left(S-\delta n^{2} H^{2}\right)_{+}^{n / 2} d M \geq c(n, \delta) \sum_{i=k}^{n-k} \tau_{i}(f) \\
& \geq c(n, \delta) \sum_{i=k}^{n-k} \beta_{i}(M ; \mathbb{F}),
\end{array}
$$

where

$$
c(n, \delta)=\min _{2 \leq k \leq n / 2}\left\{2\left(\frac{\varepsilon(n, k, \delta)}{2}\right)^{n / 4} \operatorname{Vol}\left(\mathbb{S}^{n+k-1}\right)\right\} .
$$


Now, assume that

$$
\int_{M^{n}}\left\|R-\frac{\mathrm{scal}}{n(n-1)} R_{1}\right\|^{n / 2} d M+\int_{M^{n}}\left(S-\delta n^{2} H^{2}\right)_{+}^{n / 2} d M<c(n, \delta) .
$$

Then it follows directly from $(22)$ that $\sum_{i=k}^{n-k} \tau_{i}(f)<1$. Thus, there exists $u \in \mathbb{S}^{n-k-1}$ such that the height function $h_{u}: M^{n} \rightarrow \mathbb{R}$ is a Morse function whose number of critical points of index $i$ satisfies $\mu_{i}(u)=0$ for any $k \leq i \leq n-k$. The fundamental theorem of Morse theory (cf. [17, Theorem 3.5] or [5, Theorem 4.10]) then implies that $M^{n}$ has the homotopy type of a CW-complex with no cells of dimension $i$ for $k \leq i \leq n-k$.

Now, if $k=2$, there will be no 2-cells and thus by the cellular approximation theorem we conclude that the inclusion of the 1-skeleton $\mathrm{X}^{(1)} \hookrightarrow M^{n}$ induces isomorphism between the fundamental groups. Therefore, the fundamental group $\pi_{1}\left(M^{n}\right)$ is a free group on $\beta_{1}\left(M^{n} ; \mathbb{Z}\right)$ elements and $H_{1}\left(M^{n} ; \mathbb{Z}\right)$ is a free abelian group on $\beta_{1}\left(M^{n} ; \mathbb{Z}\right)$ generators. In particular, if $\pi_{1}\left(M^{n}\right)$ is finite, then $\pi_{1}\left(M^{n}\right)=0$ and hence $H_{1}\left(M^{n} ; \mathbb{Z}\right)=0$. From Poincaré duality and the universal coefficient theorem it follows that $H_{n-1}\left(M^{n} ; \mathbb{Z}\right)=0$. Thus, $M^{n}$ is a simply connected homology sphere and hence a homotopy sphere. By the generalized Poincaré conjecture (Smale $n \geq 5$, Freedman $n=4$ ) we deduce that $M^{n}$ is homeomorphic to $\mathbb{S}^{n}$.

If the scalar curvature is everywhere non-positive, then from the Gauss equation we obtain $S \geq \delta n^{2} H^{2}$. Using Proposition 9 and the Gauss equation we have

$$
\left(\left\|R-\frac{\mathrm{scal}}{n(n-1)} R_{1}\right\|^{2}+\left(S-\delta n^{2} H^{2}\right)^{2}\right)^{n / 4}(p) \geq(\varepsilon(n, k, \delta))^{n / 4} \int_{\mathbb{S}_{p}^{k-1}}\left|\operatorname{det} A_{\xi}\right| d V_{\xi}
$$

for all $p \in M^{n}$. Integrating over $M^{n}$, we obtain

$$
\int_{M^{n}}\left(\left\|R-\frac{\mathrm{scal}}{n(n-1)} R_{1}\right\|^{2}+\left(S-\delta n^{2} H^{2}\right)^{2}\right)^{n / 4} d M \geq(\varepsilon(n, k, \delta))^{n / 4} \int_{U N_{f}}\left|\operatorname{det} A_{\xi}\right| d \Sigma .
$$

Bearing in mind the definition of the total absolute curvature $\tau(f)$ of $f$ and (21), we have

$$
\begin{gathered}
\int_{M^{n}}\left\|R-\frac{\mathrm{scal}}{n(n-1)} R_{1}\right\|^{n / 2} d M+\int_{M^{n}}\left(S-\delta n^{2} H^{2}\right)^{n / 2} d M \\
\geq 2(\varepsilon(n, k, \delta) / 2)^{n / 4} \operatorname{Vol}\left(\mathbb{S}^{n+k-1}\right) \tau(f) \geq c(n, \delta) \tau(f) .
\end{gathered}
$$

The desired inequality follows from Theorem 14 .

If the scalar curvature is everywhere non-positive and

$$
\int_{M^{n}}\left\|R-\frac{\text { scal }}{n(n-1)} R_{1}\right\|^{n / 2} d M+\int_{M^{n}}\left(S-\delta n^{2} H^{2}\right)^{n / 2} d M<3 c(n, \delta)
$$

then from $(23)$ we obtain $\tau(f)<3$. This implies that there exists a height function which is a Morse function with exactly two critical points. Reeb's theorem then implies that $M^{n}$ is homeomorphic to $\mathbb{S}^{n}$. 
Proof of Corollary 3: Our assumptions and Theorem 1 imply that $\beta_{i}\left(M^{n} ; \mathbb{F}\right)=0, k \leq i \leq$ $n-k$. Hence, $f$ is $\delta$-pinched and the rest of the proof follows from Theorem 1. Moreover, if the mean curvature is everywhere positive and $f$ is $1 /(n-1)$-pinched, then a result due to Andrews and Baker [1] implies that $M^{n}$ is diffeomorphic to $\mathbb{S}^{n}$.

Proof of Corollary 4: Our assumptions and Theorem 1 imply $R=(\operatorname{scal} / n(n-1)) R_{1}$. It follows from Shur's lemma that $M^{n}$ is a space form. According to a result due to Chern, Otsuki and Kuiper (cf. [15, Corollary 4.8]) the sectional curvature must be positive. Appealing to Moore [18, Proposition 4], $M^{n}$ is isometric to a constant curvature sphere.

Proof of Corollary 5: We consider the immersion $\tilde{f}=i \circ f$, where $i$ : $\mathbb{S}^{n+k-1} \hookrightarrow \mathbb{R}^{n+k}$ is the totally umbilic inclusion and the proof follows directly from Theorem 1.

Proof of Theorem 6: Let $f:\left(M^{n}, g\right) \rightarrow \mathbb{R}^{n+k}$ be a conformal immersion with second fundamental form $\alpha$ and shape operator $A_{\xi}$ with respect to $\xi$, where $(p, \xi) \in U N_{f}$. Using the Gauss equation, it follows that the Weyl tensor $\mathcal{W}_{\tilde{g}}$ with respect to the induced metric $\tilde{g}$ of $f$ is given by $\mathcal{W}_{\tilde{g}}(p)=\mathbf{W}(\alpha(p))$. From Proposition 12, we have

$$
\left(\left\|\mathcal{W}_{\tilde{g}}\right\|^{2}+\left(S-\delta n^{2} H^{2}\right)_{+}^{2}\right)^{n / 4}(p) \geq\left(\varepsilon_{1}(n, k, \delta)\right)^{n / 4} \int_{\Omega(\alpha(p))}\left|\operatorname{det} A_{\xi}\right| d V_{\xi}
$$

for all $p \in M^{n}$. By integrating over $M^{n}$ and using (19), we obtain

$$
\int_{M^{n}}\left(\left\|\mathcal{W}_{\tilde{g}}\right\|^{2}+\left(S-\delta n^{2} H^{2}\right)_{+}^{2}\right)^{n / 4} d M \geq\left(\varepsilon_{1}(n, k, \delta)\right)^{n / 4} \operatorname{Vol}\left(\mathbb{S}^{n+k-1}\right) \sum_{i=k+1}^{n-k-1} \tau_{i}(f) .
$$

Observe that

$$
\left(\left\|\mathcal{W}_{\tilde{g}}\right\|^{2}+\left(S-\delta n^{2} H^{2}\right)_{+}^{2}\right)^{n / 4}(p) \leq 2^{(n-4) / 4}\left(\left\|\mathcal{W}_{\tilde{g}}\right\|^{n / 2}+\left(S-\delta n^{2} H^{2}\right)_{+}^{n / 2}\right)(p)
$$

for all $p \in M^{n}$. Thus, from (24), (18) and the fact that the $L^{n / 2}$-norm of the Weyl tensor is conformally invariant, we have that

$$
\int_{M^{n}}\|\mathcal{W}\|^{n / 2} d M+\int_{M^{n}}\left(S-\delta n^{2} H^{2}\right)_{+}^{n / 2} d M \geq c_{1}(n, \delta) \sum_{i=k+1}^{n-k-1} \tau_{i}(f) \geq c_{1}(n, \delta) \sum_{i=k+1}^{n-k-1} \beta_{i}\left(M^{n} ; \mathbb{F}\right)
$$

where

$$
c_{1}(n, \delta)=\min _{2 \leq k \leq[(n-2) / 2]}\left\{2\left(\frac{\varepsilon_{1}(n, k, \delta)}{2}\right)^{n / 4} \operatorname{Vol}\left(\mathbb{S}^{n+k-1}\right)\right\} .
$$

Now, assume that

$$
\int_{M^{n}}\|\mathcal{W}\|^{n / 2} d M+\int_{M^{n}}\left(S-\delta n^{2} H^{2}\right)_{+}^{n / 2} d M<c_{1}(n, \delta) .
$$


Then it follows from $(25)$ that $\sum_{i=k+1}^{n-k-1} \tau_{i}(f)<1$. Thus, there exists $u \in \mathbb{S}^{n+k-1}$ such that the height function $h_{u}: M^{n} \rightarrow \mathbb{R}$ is a Morse function whose number of critical points of index $i$ satisfies $\mu_{i}(u)=0$ for any $k<i<n-k$. The fundamental theorem of Morse theory then implies that $M^{n}$ has the homotopy type of a CW-complex with no cells of dimension $i$ for $k<i<n-k$.

\section{References}

[1] B. Andrews and C. Baker, Mean curvature flow of pinched submanifolds to spheres, J. Differential Geom. 85 (2010), no. 3, 357-395. MR2739807

[2] K.O. Araújo and K. Tenenblat, On submanifolds with parallel mean curvature vector, Kodai Math. J. 32 (2009), no. 1, 59-76, DOI 10.2996/kmj/1238594546. MR2518554

[3] E.R. Barbosa and K.O. Araújo, On complete submanifolds with bounded mean curvature, J. Geom. Phys. 61 (2011), no. 10, 1957-1964, DOI 10.1016/j.geomphys.2011.05.005. MR2822462

[4] E. Cartan, La déformation des hypersurfaces dans l'espace conforme réel à $n \geq 5$ dimensions, Bull. Soc. Math. France 45 (1917), 57-121 (French). MR1504762

[5] J. Cheeger and D.G. Ebin, Comparison theorems in Riemannian geometry, North-Holland Publishing Co., Amsterdam-Oxford; American Elsevier Publishing Co., Inc., New York, 1975. North-Holland Mathematical Library, Vol. 9. MR0458335

[6] B.Y. Chen and M. Okumura, Scalar curvature, inequality and submanifold, Proc. Amer. Math. Soc. 38 (1973), 605-608. MR0343217

[7] Q.M. Cheng, Topology and geometry of complete submanifolds in Euclidean spaces, PDEs, submanifolds and affine differential geometry, Banach Center Publ., vol. 69, Polish Acad. Sci., Warsaw, 2005, pp. $67-80$, DOI 10.4064/bc69-0-3, (to appear in print). MR2188755

[8] Q.M. Cheng and K. Nonaka, Complete submanifolds in Euclidean spaces with parallel mean curvature vector, Manuscripta Math. 105 (2001), no. 3, 353-366, DOI 10.1007/s002290100186. MR1856616

[9] S.S. Chern, M. do Carmo, and S. Kobayashi, Minimal submanifolds of a sphere with second fundamental form of constant length, Functional Analysis and Related Fields (Proc. Conf. for M. Stone, Univ. Chicago, Chicago, Ill., 1968), Springer, New York, 1970, pp. 59-75. MR0273546

[10] S.S. Chern and N.H. Kuiper, Some theorems on the isometric imbedding of compact Riemann manifolds in euclidean space, Ann. of Math. (2) 56 (1952), 422-430. MR0050962

[11] S.S. Chern and R.K. Lashof, On the total curvature of immersed manifolds, Amer. J. Math. 79 (1957), 306-318. MR0084811

[12] _ On the total curvature of immersed manifolds. II, Michigan Math. J. 5 (1958), 5-12. MR0097834

[13] M. do Carmo, M. Dajczer, and F. Mercuri, Compact conformally flat hypersurfaces, Trans. Amer. Math. Soc. 288 (1985), no. 1, 189-203, DOI 10.2307/2000435. MR773056

[14] N.H. Kuiper, Minimal total absolute curvature for immersions, Invent. Math. 10 (1970), 209-238. MR0267597

[15] S. Kobayashi and K. Nomizu, Foundations of differential geometry. Vol. II, Interscience Tracts in Pure and Applied Mathematics, No. 15 Vol. II, Interscience Publishers John Wiley \& Sons, Inc., New York-London-Sydney, 1969. MR0238225 
[16] P.F. Leung, Minimal submanifolds in a sphere, Math. Z. 183 (1983), no. 1, 75-86, DOI 10.1007/BF01187216. MR701359

[17] J. Milnor, Morse theory, Based on lecture notes by M. Spivak and R. Wells. Annals of Mathematics Studies, No. 51, Princeton University Press, Princeton, N.J., 1963. MR0163331

[18] J.D. Moore, Submanifolds of constant positive curvature. I, Duke Math. J. 44 (1977), no. 2, $449-484$. MR0438256

[19] _ Conformally flat submanifolds of Euclidean space, Math. Ann. 225 (1977), no. 1, 89-97. MR0431046

[20] Codimension two submanifolds of positive curvature, Proc. Amer. Math. Soc. 70 (1978), no. 1, 72-74, DOI 10.2307/2042587. MR487560

[21] Euler characters and submanifolds of constant positive curvature, Trans. Amer. Math. Soc. 354 (2002), no. 9, 3815-3834 (electronic), DOI 10.1090/S0002-9947-02-03043-X. MR1911523

[22] C.R. Onti and Th. Vlachos, Almost conformally flat hypersurfaces, Arxiv e-prints (2016), available at http://arxiv.org/abs/1610.07349

[23] T. Otsuki, On the existence of solutions of a system of quadratic equations and its geometrical application, Proc. Japan Acad. 29 (1953), 99-100. MR0060281

[24] K. Shiohama, Sphere theorems, Handbook of differential geometry, Vol. I, North-Holland, Amsterdam, 2000, pp. 865-903, DOI 10.1016/S1874-5741(00)80011-4, (to appear in print). MR1736859

[25] K. Shiohama and H. Xu, Lower bound for $L^{n / 2}$ curvature norm and its application, J. Geom. Anal. 7 (1997), no. 3, 377-386, DOI 10.1007/BF02921626. MR1674797

[26] _ Rigidity and sphere theorems for submanifolds, Kyushu J. Math. 48 (1994), no. 2, 291-306, DOI 10.2206/kyushujm.48.291. MR1294532

[27] _ Rigidity and sphere theorems for submanifolds. II, Kyushu J. Math. 54 (2000), no. 1, 103109, DOI 10.2206/kyushujm.54.103. MR1762795

[28] J. Simons, Minimal varieties in riemannian manifolds, Ann. of Math. (2) 88 (1968), 62-105. MR0233295

[29] K. Smoczyk, Mean Curvature Flow in higher codimension: introduction and survey. Global differential geometry, Springer Proc. Math., vol. 17, Springer, Heidelberg, 2012, pp. 231-274

[30] Th. Vlachos, Integral curvature and topological obstructions for submanifolds, Geom. Dedicata 166 (2013), 289-305, DOI 10.1007/s10711-012-9796-z. MR3101171

Department of Mathematics, University of IoAnnina, 45110 IoAnnina, Greece E-MAIL ADRRESSES: tvlachos@uoi.gr, chonti@cc.uoi.gr 